\title{
Surrogates under scrutiny: fallible correlations, fatal consequences
}

\author{
We need a new approach to proxy measures of health, says Ray Moynihan
}

\author{
Ray Moynihan conjoint lecturer
}

University of Newcastle, Australia

We live in a time when much disease is measured not by symptoms but by numbers, determined by biomarkers in our blood or bone. ${ }^{1}$ Transforming a healthy person's risk of disease into a chronic condition has been a key characteristic of modern medicine, creating vast new markets for "preventive" pills designed to reduce suffering and extend life. The annual global spend on cholesterol lowering drugs alone has exceeded $£ 10$ bn ( $€ 11 \mathrm{bn} ; \$ 16 \mathrm{bn}$ ), while more generally widening definitions and lowering thresholds continue to expand the patient pool. ${ }^{2}$ Well funded campaigns urge the public to know their numbers, and professionals are rewarded for treating to target. Yet the grand assumption underpinning this approach —-that helping a person's numbers will automatically improve their health-is a delusion as dangerous as it is seductive.

Use of flecainide to reduce the number of irregular heart beats, for some people also raised their risk of an early death, killing tens of thousands just decades ago. ${ }^{34}$ Long term hormone replacement therapy lowered "bad" cholesterol and raised "good" cholesterol for generations of women, but it also lifted their chances of heart attacks and strokes. ${ }^{5}$ Prescribing pills to aggressively decrease blood sugar in high risk diabetes patients has been increasing their risks of disease and premature death rather than reducing them. ${ }^{6}$ Yet decisions to approve and prescribe drugs based on success with surrogate end points continue apace, as do their sometimes deadly consequences.

Since at least the 1950s studies showing correlations like those between high blood pressure and heart disease have led us to believe that if we can modify people's biomarkers, we can lower their risks of disease or early death. While the theory sometimes works, its logical flaw is obvious. Whether we help or harm depends on how we try to lower risks - and long term treatments often carry unintended consequences. Moreover, even when significant clinical benefit is proved, the often minimal risk reductions associated with long term treatment suggest that the current approach may be over-medicalising many for little gain and at great cost.

\section{Unproved benefits}

"I think we've been far too cavalier in accepting favourable changes in a biomarker as a perfect proxy for patient benefit," says Yale University public health professor Harlan Krumholz, who helped put together a recent report on surrogates for the United States Institute of Medicine. ${ }^{7}$ He says the focus on "knowing your numbers" and "treating to target" seems to play to everyone's best interest-they are easy public health messages, they need only quick visits to doctors' offices, and are a great boon for companies, which don't have to do the larger long term studies of effects on clinically meaningful outcomes. Professor Krumholz believes the evidence shows "simple assumptions" about surrogates are often incorrect, and he argues we need to better inform people about potential dangers. "If a drug is approved only on the evidence of its impacts on a biomarker, there should be big clear warnings saying it has an unproven effect on patient health," he says. "We need to convey the uncertainty."

That recent report from the Institute of Medicine-Evaluation of Biomarkers and Surrogate Endpoints in Chronic Disease-is sobering reading. ${ }^{7}$ It details an often misplaced confidence in relying on surrogates to assess treatment benefits. "Remarkably, the cautionary voices speaking about the risks of using surrogate endpoints have been repeating the same messages for 20 years," says the report. "What has been changing is the continually increasing amount of data supporting their arguments." The report cites examples where treatments have benefited surrogate measures but harmed people, and it urges a far more rigorous evaluation of how these intermediate end points are used.

Even for widely used surrogates there's more uncertainty than we might imagine. Although blood pressure is an extremely reliable proxy, questions arise from the fact that treatments similarly affecting blood pressure can have different effects on heart disease. ${ }^{7}$ In the face of the AIDS crisis, researchers discovered HIV-1 RNA was a valuable biomarker, enabling experimental drugs to be assessed quickly. However, in some 
contexts, short term changes in that biomarker proved to be a poor surrogate because partial suppression of the virus allowed the formation of drug resistant mutations and limited future usefulness of drugs. ${ }^{7}$ And although reduction in tumour size is sometimes a useful way to measure the effect of cancer treatment, even major shrinkage does not always represent meaningful improvement because, for example, in some cancers, smaller tumours tend to grow faster than larger ones. ${ }^{7}$ There are even doubts about the value of cholesterol, and it serves as a powerful case study of the need for a new way of thinking about surrogate markers.

\section{Caution over cholesterol}

Regulators, including the United States Food and Drug Administration, have officially sanctioned low density lipoprotein (LDL) cholesterol as a surrogate end point, allowing many drugs to be approved and marketed if they reduce this "bad cholesterol" without requiring proof they actually improve health. Yet despite the size of the global markets, there are myriad uncertainties surrounding cholesterol. According to the 2010 Institute of Medicine report, although the methods used to determine blood cholesterol are reliable and reproducible, they do not directly measure LDL cholesterol and so have "limitations." In addition, though LDL cholesterol is "hypothesised" to have a causal role in the atherosclerotic disease process, it has "not been conclusively proven."7 And reinforcing the general point about our over-confidence in surrogates, the report reminds us that lowering LDL cholesterol "does not always correlate with improved patient outcome." The report points out there are over 200 coronary risk factors, and that cholesterol, while currently considered by many to be a valuable biomarker for heart disease, is only one of "multiple determinants" and "numerous other mediators."

The benefits of long term preventive therapies like cholesterol lowering drugs are usually portrayed as relative reductions in risk, but when the risks are considered in absolute terms, a different picture emerges. For example, based on a Cochrane review of trials for primary prevention, ${ }^{8}$ there has been recent enthusiasm that for people without a history of heart disease statins can reduce premature deaths by $17 \%$, coronary heart disease by $28 \%$, strokes by $22 \%$, and revascularisation by $34 \% .{ }^{9}$ Yet a close reading of the tables from that systematic review suggests the estimated absolute risk reductions with around four to five years of drug taking are $0.5 \%$ for death, $1.9 \%$ for coronary heart disease, $0.5 \%$ for stroke, and $0.7 \%$ for revascularisation. ${ }^{8}$ The estimated number needed to treat for four to five years thus ranges from 50 to 200 depending on the outcome measure. So according to this evidence, most people taking long term statins for primary prevention gain no direct benefit.

While there is strong evidence of benefit, reinforced in other recent studies, ${ }^{10}$ the magnitude of that benefit in absolute terms is extremely small for those at low risk. There is also a question over the effects of industry funding on this evidence, as even a small amount of bias in the original trials could make the difference between an overall finding of benefit or an overall finding of no effect, in the systematic review's summary. In fact, the Cochrane reviewers' discussion of their results offers cause for great caution in interpreting the existing evidence about statins for primary prevention, and reinforces wider questions about the benefits of long term preventive medicines for otherwise healthy people.

The reviewers said they were unable to disaggregate composite outcome measures reported in the 14 statin trials they reviewed; that one third of those original trials reported outcomes selectively; and a majority did not even report on the drugs' possible harms. Two of the larger cholesterol lowering trials included for review were stopped prematurely because of statistically significant benefits being achieved early, which "may lead to an over-estimation of treatment effects." The Cochrane review also reported that all but one of the original trials had some form of drug company sponsorship, which has been shown to increase the likelihood of bias favouring the sponsor's drug. "In primary prevention where world-wide the numbers of patients eligible for treatment are massive," the reviewers wrote, "there might be motivations to use composite outcomes and early stopping to get results that clearly support intervention." When an independent Canadian group recently reviewed similar evidence, they found that while these drugs undoubtedly lower cholesterol, "the claimed mortality benefit of statins for primary prevention is more likely a measure of bias than a real effect." 11

Related questions surround other commonly used surrogates which form the basis for profitable markets in drugs for people at risk of future events. A National Institutes of Health consensus conference on hepatitis B found in 2008 that while drugs improved virological or biochemical markers, there was only "low quality evidence" showing correlations between these intermediate outcomes and real world clinical outcomes, and "no conclusive evidence" that treatments reduced the risk of liver disease or death. ${ }^{12}$ The surrogate of bone mineral density has been controversial for many years, regarded by some researchers as accounting for only a minor proportion of a person's overall risk of fracture. ${ }^{13}$ And while the search continues for reliable biomarkers to enable the prevention of Alzheimer's, a recent conference found, "firm conclusions cannot be drawn about the association of any modifiable risk factor with cognitive decline or Alzheimer's disease." ${ }^{14}$ With type 2 diabetes, heavily promoted drug strategies to aggressively reduce blood sugar have raised people's risks of heart disease and death, ${ }^{15}{ }^{16}$ rather than lowered them, despite evidence that lifestyle changes are cheap and effective. ${ }^{17}$ Some argue the treatment of type 2 diabetes is a classic example of the medical profession ignoring unfavourable evidence that shows no benefit on meaningful outcomes, while favouring studies that show positive effects on surrogate numbers. ${ }^{18}$

\section{History shows drugs help make diseases}

Jeremy Greene, historian of science at Harvard University, has described "a radical restructuring of the normal and the pathological" that emerged in the second half of the 20th century as symptomless people at risk of future disease were increasingly classified as having medical conditions. ${ }^{1}$ In Prescribing by Numbers, Dr Greene charts how drug companies and their latest products have helped to shape and expand these new risk based conditions - including high blood pressure, type 2 diabetes, and high cholesterol: "Pharmaceuticals played a central and active role in the definition of these categories of illness," he writes. Dr Greene argues this process of expanding categories to include people previously considered healthy can be seen as medicalisation. But rather than a monolithic or concerted strategy driven from the boardrooms of companies or professional medical associations, it demonstrates the "porous relationship between the science and the business of health care," and the process carries benefits as well as risks.

That porous relationship between science and business is still evident in the more recent development of "quality measures," which urge doctors to test for and treat the risk based conditions 
of the healthy. In the United States one of the main agencies developing quality measures, the National Committee for Quality Assurance, is directly funded by several drug companies, along with other sponsors. ${ }^{19}$ In the United Kingdom the process is overseen by a public institution, though there are criticisms of an overly narrow focus on numbers that can be modified by drug treatment. ${ }^{20} \mathrm{~A}$ long term critic of widening boundaries of illness, Iona Heath, president of the Royal College of General Practitioners, believes many thresholds have been set far too low. She argues quality measures may be encouraging tests that create anxiety and treatments that help surrogates rather than ameliorate the suffering of people.

In recent months we have seen yet more evidence confirming the risks of our over-reliance on surrogate numbers. In May a large trial of niacin was stopped. Though combining niacin with a statin boosted so called "good" cholesterol (high density lipoprotein) high doses were also associated with a small increase in strokes. ${ }^{21}$ In June a study in JAMA uncovered a pattern of over-estimating the strength of correlations between biomarkers and their respective diseases ${ }^{22}$ highlighting the "thin line between hype and hope." 23

\section{Shift from numbers to people}

A major rethink of the role of surrogates in medicine is timely. Routinely approving and prescribing therapies on the basis of their effects on someone's numbers, rather than their health, is increasingly seen as irresponsible and dangerous. And even when evidence suggests clinical benefits of popular "preventive" medicines for those at lower risks, a rational assessment reveals many people must be treated to prevent one adverse event, so most users gain no direct benefit despite years of treatment. The cost effectiveness of this approach is unsurprisingly in doubt. ${ }^{24}$ More disturbing still are the questions about whether some of the suggested clinical benefits are real or simply artefacts of sponsorship bias.

The rigour of the evidence informed approach to medicine has in recent decades helped us all understand the limitations of relying on surrogates, and for one of its key

architects-McMaster University professor Gordon Guyatt — this problem is both historical and cultural. He argues that central to putting American medicine on a scientific basis was the assumption that an understanding of biological mechanisms would translate into improved management of patients. And while medical students over a century later are still taught to focus on fixing a person's biological numbers-whether it's cholesterol or bone density - what is urgently required is a new approach that provides genuine improvement for the person.

Understanding biological mechanisms and diagnosing by numbers has undoubtedly brought great benefits. Yet as the definitions of medical conditions relentlessly expand via that porous relationship between the science and business of healthcare, this fragmented reductionist approach is conferring multiple medical labels on vast swathes of healthy people, who are then treated with preventive drugs that won't help most of them and may hurt many. ${ }^{25}$ The magic of numbers may help corporate profits and professional pride, but at what cost to the health of ordinary people who mistake a numerical benefit for a genuine one? Surely it's time to ask if there might be a healthier new model for medicine based on far less harmful and costly ways to try to reduce human suffering.

Competing interests: The author has completed the ICJME unified disclosure form at www.icmje.org/coi_disclosure.pdf (available on request from him) and declares no support from any organisation for the submitted work; no financial relationships with any organisation that might have an interest in the submitted work in the previous three years; and no other relationships or activities that could appear to have influenced the submitted work.

Provenance and peer review: Commissioned; externally peer reviewed.

1 Greene J. Prescribing by numbers; drugs and the definition of disease. Johns Hopkins University Press, 2007.

2 Moynihan R. A new deal on disease definition. BMJ 2011;342:d2548.

3 Cardiac Arrhythmia Suppression Trial (CAST) Investigators. Preliminary report: effect of encainide and flecainide on mortality in a randomized trial of arrhythmia suppression after myocardial infarction. N Engl J Med 1989;321:406-12.

4 Moore T. Deadly medicine. Simon and Shuster, 1995

5 Writing Group for the Women's Health Initiative Investigators. Risks and benefits of estrogen plus progestin in healthy postmenopausal women. JAMA 2002;288:321-33.

6 ACCORD Study Group. Long-term effects of intensive glucose lowering on cardiovascular outcomes. N Engl J Med 2011;364:818-28.

7 Micheel CM, Ball JR, eds. Institute of Medicine. Evaluation of biomarkers and surrogate endpoints in chronic disease. National Academies Press, 2010.

8 Taylor F, Ward K, Moore THM, Burke M, Davey Smith G, Casas JP, Ebrahim S. Statins for the primary prevention of cardiovascular disease. Cochrane Database Syst Rev 2011;1:CD004816.

9 Deckers J, Blumenthal R. Statins for primary prevention of cardiovascular disease. BMJ 2011;342:d1048.

10 Cholesterol Treatment Trialists' (CTT) Collaboration. Efficacy and safety of more intensive lowering of LDL cholesterol: a meta-analysis of data from 170000 participants in 26 randomised trials. Lancet 2010;376:1670-81.

11 Do statins have a role in primary prevention? An update. Therapeutics Letter 2010;77. www.ti.ubc.ca/letter77.

$12 \mathrm{NIH}$ Consensus Development Conference Management of Hepatitis B, Final statement 2008. http://consensus.nih.gov/2008/hepb.htm.

13 De Laet C, van Hout B, Burger H, Hofman A, Pols H. Bone density and risk of hip fracture in men and women: cross sectional analysis. BMJ 1997;351:221-5.

14 Preventing Alzheimer's disease and cognitive decline, 2010. http://consensus.nih.gov/ 2010/alzstatement.htm.

15 ACCORD Study Group. Long term effects of intensive glucose lowering on cardiovascular outcomes. N Engl J Med 2011:364:818-28.

16 Yudkin JS, Richter B, Gale EAM. Intensified glucose control in type 2 diabetes-whose agenda? Lancet 2010;377:1220-2.

17 Montori V, Isley W, Guyatt G. Waking up from the DREAM of preventing diabetes with drugs. BMJ 2007;334:882-4.

18 Shaughnessy A, Slawson D, Lewis Barnett B. What happened to the valid POEMs? A survey of review articles on the treatment of type 2 diabetes. BMJ 2003;327:266.

19 Rose J. Industry influence in the creation of pay-for-performance quality measures. Qual Manage Health Care 2003;17: 27-34.

20 Heath I, Hippisley-Cox J, Smeeth L. Measuring performance and missing the point. BMJ 2007;335;1075-6.

21 NIH stops clinical trial on combination cholesterol treatment. NIH News 2011 May 26. www.nih.gov/news/health/may2011/nhlbi-26.htm.

22 Ioannidis JPA, Orestis A, Panagiotou MD. Comparison of effect sizes associated with biomarkers reported in highly cited individual articles and in subsequent meta-analyses. JAMA 2011;305:2200-10.

23 Bossuyt $\mathrm{P}$. The thin line between hope and hype in biomarker research. JAMA 2011;305:2229-30

24 Järvinen $\mathrm{T}$, Sievänen $\mathrm{H}$, Kannus $\mathrm{P}$, Jokihaara J, Khan $\mathrm{K}$. The true cost of pharmacological disease prevention. BMJ 2011;342:d2175.

25 Starfield B, Hyde J, Gervas J, Heath I. The concept of prevention: a good idea gone astray? J Epidemiol Community Health 2008;62:580-3.

Cite this as: BMJ 2011;343:d5160 